\title{
Construction of a Brain Template from MR Images Using State-of-the-Art Registration and Segmentation Techniques
}

\author{
Dieter Seghers, Emiliano D’Agostino, Frederik Maes`, Dirk Vandermeulen, and \\ Paul Suetens
}

\begin{abstract}
Katholieke Universiteit Leuven, Faculties of Medicine and Engineering, Medical Image Computing (Radiology - ESAT/PSI), University Hospital Gasthuisberg, Herestraat 49, B-3000 Leuven, Belgium

dieter.seghers@uz.kuleuven.ac.be
\end{abstract}

\begin{abstract}
We propose a procedure for generating a brain atlas with mean morphology and mean intensity by state-of-the-art non-rigid registration of a database of MR images of normal brains. The new constructed atlas is much sharper that currently available linear atlases, as the residual inter-subject shape variability after both linear and subsequent non-linear normalization is retained. As a consequence, the resulting atlas is suited as a mean shape template for brain morphometry approaches that are based on non-rigid atlas-to-subject image registration.
\end{abstract}

\section{Introduction}

A brain atlas is an important tool in the processing of images of the brain. It contains prior knowledge about the human brain that is useful for the segmentation and registration of a new brain image. The construction of an atlas from a database of subject images consists of a spatial-normalization step and usually also an intensity-normalization step. The spatial normalization is needed to construct an averaged atlas image whith a mean morphology, while intensity normalization avoids that the intensities in the atlas image are dominated by a single subject image.

A widely used atlas of the human brain is the MNI atlas, that is the standard atlas template in SPM 1]. This atlas was constructed using spatial normalization by linear registration with 9 degrees of freedom. Linear registration does not comenpsate for local shape differences in the brain, which induces blurring, not only in the averaged MR template but also in the tissue distribution maps obtained by averaging segmentations of white matter (WM), gray matter (GM) and cerebrospinal fluid (CSF) over all subjects. This makes linear atlases not suited as a mean shape template for brain morphometry approaches that are

\footnotetext{
* Frederik Maes is Postdoctoral Fellow of the Fund for Scientific Research - Flanders (FWO-Vlaanderen, Belgium).
} 
based on non-rigid atlas-to-subject image registration. Hence, there is need for adequate brain atlases constructed by appropriate non-rigid spatial normalization.

In this paper we present a procedure for generating a brain atlas with mean morphology and mean intensity by state-of-the-art non-rigid registration of a database of MR images of normal brains. Similar to the approach of Guimond [2], all images in the database are aligned with a single template image. All images in the database are in turn selected as template image and subsequently deformed towards the mean shape of all other non-rigidly aligned images to eliminate bias in the atlas towards any of the original images in the database. Average segmentation maps for the distribution of WM, GM and CSF are also constructed from the intensity-based tissue classification maps of the original images. The resulting atlas is much sharper than currently available linear atlases, as only the residual inter-subject shape variability after both linear and subsequent nonlinear normalization is retained.

\section{Materials and Methods}

\subsection{Image Database}

The atlas is constructed from a database of MR images of normal brains. In this paper, a database of $N=64$ images was used. The subjects were 41 males and 23 females, aged 22 to 40. All images were acquired using a 3D MPRAGE sequence with sagittal orientation. The images have dimensions $160 \times 256 \times 256$ with a voxelsize of $1 \times 1 \times 1 \mathrm{~mm}^{3}$.

\subsection{Image Preprocessing}

Segmentation and masking. A first step in the construction of the brain atlas is the masking of non-brain tissues in the images in the database. The images are therefore first segmented using the model-based tissue classification algorithm of Van Leemput et al [4] to obtain probabilistic WM, GM and CSF maps. A brain mask is created by summing these maps and thresholding at $50 \%$ probability. Figure 1 shows the result of this procedure for a particular brain.

Affine registration. Pose and size related differences in the position, orientation and scale of the brains in the images in the database are eliminated prior to atlas construction by transforming all images into the same space by a 12 parameter affine transformation. All images in the database are therefore affinely registered to the template MR image distributed with SPM99 [1] by maximization of mutual information [3].

\subsection{Atlas Construction}

Nonrigid registration. The key in the proposed procedure for atlas construction is non-rigid voxel-based image registration. Non-rigid registration finds the 


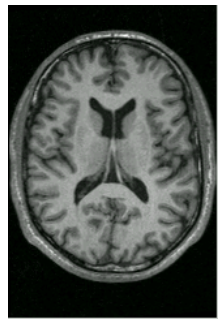

(a)

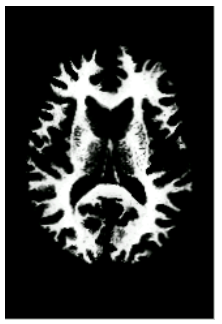

(b)

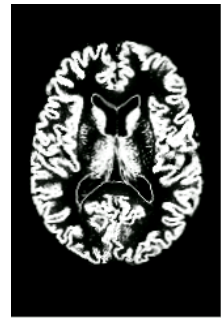

(c)

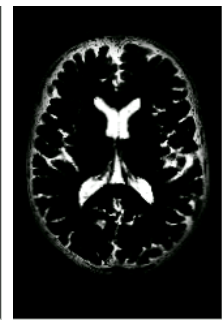

(d)

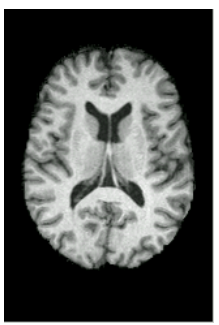

(e)

Fig. 1. The brain image is masked by first segmenting the image using the EMS algorithm of Van Leemput et al [4, which delivers probabilistic WM, GM and CSF maps. The sum of those maps serves as a mask to the brain image. From left to right: (a) the original image, its maps for (b) WM, (c) GM and (d) CSF and (e) the masked image.

deformation field $T(x, y, z): \mathbb{R}^{3} \rightarrow \mathbb{R}^{3}$ that maps coordinates (x,y,z) in the reference image $\mathrm{R}$ into coordinates $\left(x^{\prime}, y^{\prime}, z^{\prime}\right)$ in the floating image $\mathrm{F}$ :

$$
\left(x^{\prime}, y^{\prime}, z^{\prime}\right)=\left(x-T_{x}(x, y, z), y-T_{y}(x, y, z), z-T_{z}(x, y, z)\right)
$$

The warped image $F^{\prime}$ is computed as:

$$
F^{\prime}(x, y, z)=F\left(x-T_{x}(x, y, z), y-T_{y}(x, y, z), z-T_{z}(x, y, z)\right)
$$

which requires interpolation to evaluate $F$ at the non-integer positions $\left(x^{\prime}, y^{\prime}, z^{\prime}\right)$ (in our case, trilinear interpolation is used). To simplify notation, equation (10) will be noted as $F^{\prime}=T(F)$. The registration algorithm finds a transformation $T$ that maximizes a similarity measure between the warped image $F^{\prime}$ and the reference image, subject to appropriate regularization constraints. The method used in this work is the algorithm proposed by D'Agostino et al [5] based on maximization of mutual information between the deformed floating image and the reference image. The deforming reference image is considered as a viscous fluid whose motion is governed by the Navier-Stokes equation of conservation of momentum. This algorithm is well suited to match one MR brain image to another because it allows large deformations. The mutual information criterium is chosen in order to cope with the intensity bias fields of the subject images.

Mean morphology. The aim of atlas construction is to recover the mean morphology of the brain from a database of images, assuming that the database is representative for the population of interest. To do so, we apply the following procedure (figure 2). Let $A_{0}$ denote any particular brain in the database. We want to transform $A_{0}$ in a new image $A_{1}$ with mean morphology as observed in the database. First, all database images $I_{1}, \ldots, I_{N}$ are registered to $A_{0}\left(A_{0}\right.$ is the floating image and the database images act as reference images), yielding deformations $T_{l} \forall l=1, \ldots, N$. Consider an arbitrary point $(i, j, k)$ at the same geometrical position in the space of each of the original images $I_{l}$ (after affine 
registration as described above). This point corresponds to different anatomical locations in each image. These points are mapped by each $T_{l}$ into the space of the selected template $A_{0}$ :

$$
\left(i_{l}, j_{l}, k_{l}\right)=(i, j, k)-\left(T_{l, x}(i, j, k), T_{l, y}(i, j, k), T_{l, z}(i, j, k)\right) \quad \forall l=1, \ldots, N
$$

We consider the point at the center of these projected points as the anatomical point that on average (for our database of images) matches the geometrical point $(i, j, k)$. Hence, we define the mean morphology image $A_{1}$ as the image obtained from $A_{0}$ by the spatial transformation

$$
A_{1}(i, j, k)=A_{0}(\bar{i}, \bar{j}, \bar{k})
$$

with

$$
(\bar{i}, \bar{j}, \bar{k})=\frac{1}{N} \sum_{l=1}^{N}\left(i_{l}, j_{l}, k_{l}\right)=\left(i-\bar{T}_{x}, j-\bar{T}_{y}, k-\bar{T}_{z}\right)
$$

$\bar{T}_{x}, \bar{T}_{y}, \bar{T}_{z}$ represent the averaged sum of the deformation fields. Equation (2) becomes

$$
A_{1}(i, j, k)=A_{0}\left(i-\bar{T}_{x}(i, j, k), j-\bar{T}_{y}(i, j, k), k-\bar{T}_{z}(i, j, k)\right)
$$

which can be written as

$$
A_{1}=\bar{T}\left(A_{0}\right)
$$

In words: the particular brain is warped to each of the individuals of the database, the mean deformation field is computed and this is applied to the particular brain.

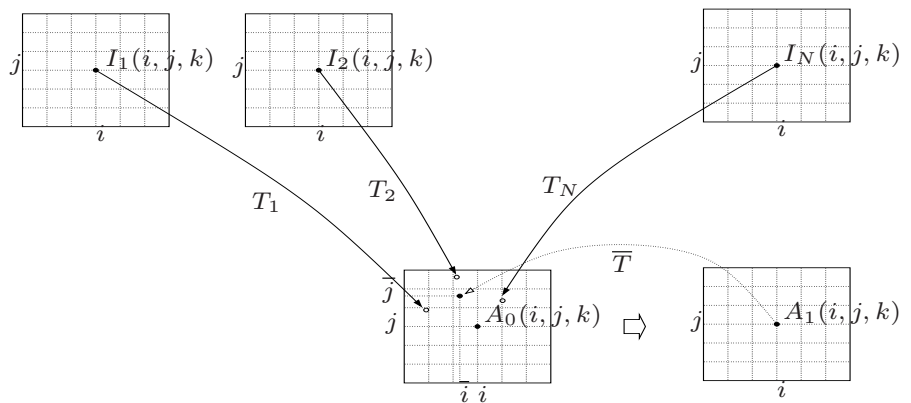

Fig. 2. A particular brain image $A_{0}$ is transformed into an image with mean morphology $A_{1}$ by the mean transformation $\bar{T}$ obtained by averaging all transformations $T_{l}$ of $A_{0}$ to each of the images $I_{l}$ in the database. 
Mean intensities. The procedure described above does not result in a suitable atlas yet, because the computed image $A_{1}$ has only intensities originating from the particular brain $A_{0}$. Moreover the topology of image $A_{1}$ will be determined by the topology of the initial image $A_{0}$, while not every brain has the same topology. To overcome these limitations, we propose the scheme presented in figure 3 Each of the database images $I_{1}, I_{2}, \ldots, I_{N}$ is in turn selected as the template $A_{0}$ and transformed into images $\bar{I}_{1}, \bar{I}_{2}, \ldots, \bar{I}_{N}$ with mean morphology using the procedure described above. This requires $N(N-1)$ non-rigid registrations in total. These mean shape images are then averaged voxel-wise after appropriate intensity rescaling to compensate for global intensity differences between the images in the database. The need for this intensity rescaling is illustrated in figure 4 To match the intensities of two images $\bar{I}_{i}$ and $\bar{I}_{j}$, linear regression between the intensities of both images is performed and the intensities in one of the images are linearly rescaled to make the slope of the regression line equal to one and its intercept equal to zero.

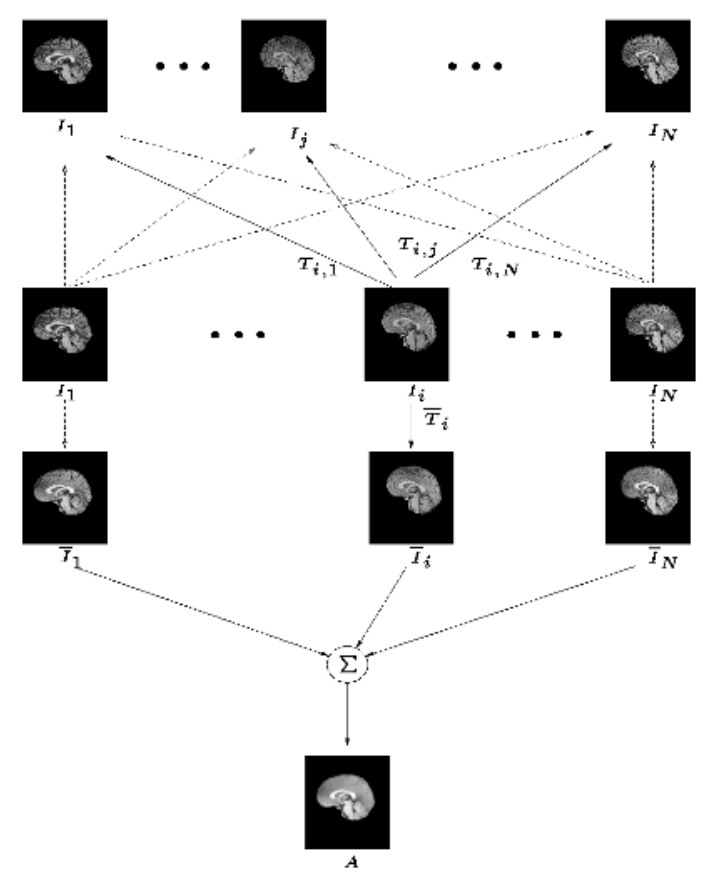

Fig. 3. Procedure to generate an atlas with mean morphology and mean intensities. Each of the images in the database is non-rigidly registered to all others and is subsequently transformed by the average deformation into a mean shape image. The mean shape images obtained for each database image are subsequently voxel-wise averaged after appropriate intensity rescaling to compensate for global intensity differences in the original images. 


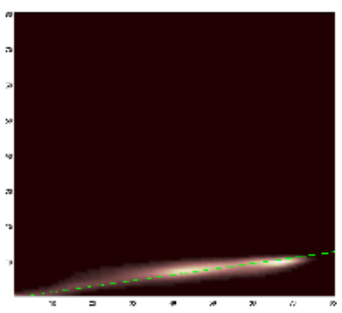

(a)

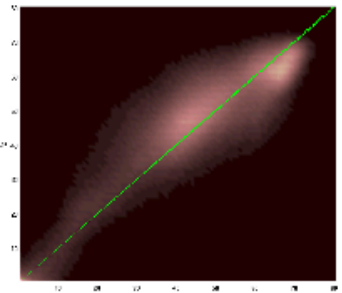

(b)

Fig. 4. (a) The joint histogram of two images $\bar{I}_{i}$ and $\bar{I}_{j}$. (b) The joint histogram of the images $\bar{I}_{i}$ and $\bar{I}_{j}$ after linear intensity rescaling to compensate for global intensity differences between both images.

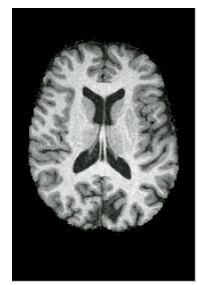

(a)

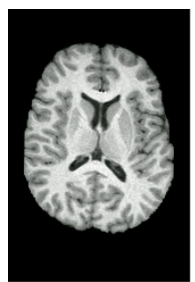

(b)

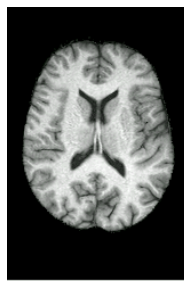

(c)

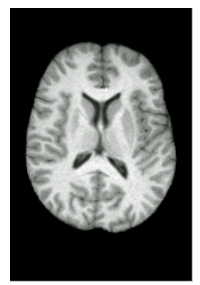

(d)

Fig. 5. Demonstration of the effect of the mean morphology transformation. (a) and (b): Two database images after preprocessing (masking and affine registration). (c) and (d): The same images but mapped into the mean morphology space. It is easy to remark that the shape of the ventricles has been normalized

Atlas with segmentation maps. Besides an atlas MR template with mean shape and gray values, we also construct the corresponding probability maps for WM, GM and CSF for use as spatially variant priors in atlas-driven intensitybased tissue classification. The segmentation maps obtained for each of the original images in the database are therefore first transformed into the mean morphology space using the already computed mean deformations $\bar{T}_{i}$ (figure 3). The projected probability maps are subsequently voxel-wise averaged.

\section{Results}

All results are computed using a database of $N=64$ normal brains. The effect of the mean morphology transformation is illustrated in figure 5 The final atlas with mean morphology and mean intensities and its corresponding segmentation maps are shown in figure 6. In figure 7 the atlas is compared with the SPM-atlas. The new atlas is obviously less smooth then the SPM-atlas. 

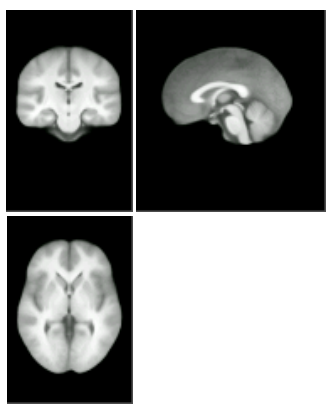

(a)
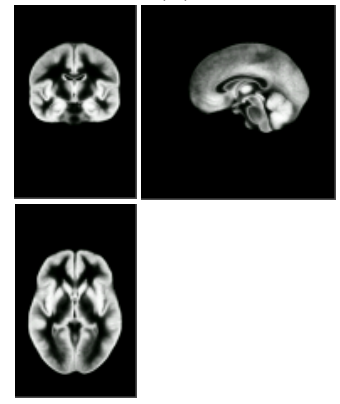
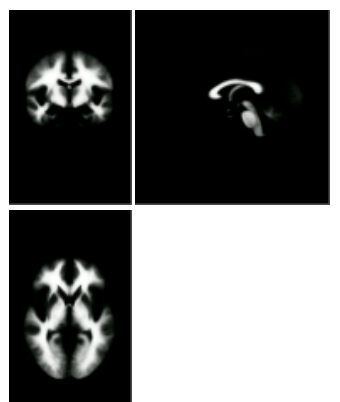

(b)

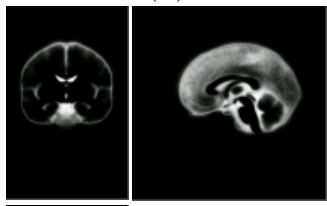

(d)

Fig. 6. Constructed atlas consisting of (a) a gray valued MR template with mean shape and intensity and corresponding probabilistic tissue distribution maps for (b) WM, (c) GM and (d) CSF.

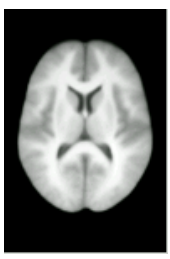

(a)

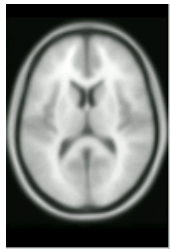

(e)

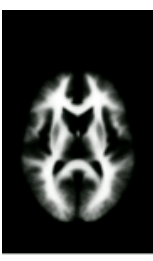

(b)

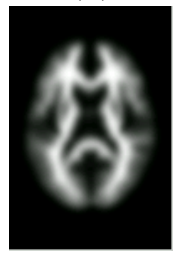

(f)

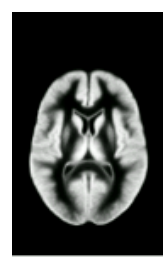

(c)

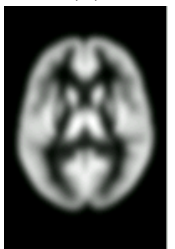

(g)

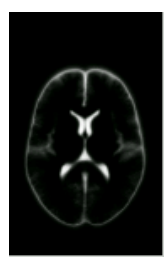

(d)

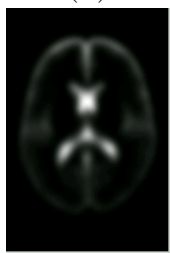

(h)

Fig. 7. Comparison of the new atlas ((a) gray values, (b) WM,(c) GM and (d) CSF) with the SPM-atlas ((e) gray values, (f) WM,(g) GM and (h) CSF). The new atlas is obviously less smooth than the SPM-atlas. 


\section{Discussion and Future Work}

The resulting atlas is well suited for applications using non-rigid atlas-to-subject image registration, as blurring in the template image is avoided by compensating for local shape differences. The presented procedure also avoids the atlas to be dominated by any of the original images in the database. Instead of groupwise registration [6], each of the original subject images is deformed towards the mean shape of all other non-rigidly aligned images. If only one of the subject images would be taken as a target image, the atlas would be biased towards the chosen target image.

A possible improvement is the expansion of the atlas-generating procedure to multiple iterations. The original database is transformed to a database of mean morphology brains. This step can be repeated till a convergence criterium is reached.

In the future, this atlas has to be compared with other brain templates using non-linear spatial normalization. An other future step, is a statistical analysis of the deformation fields that transform the single subject templates to the mean shape. Hence, inter-subject local shape variability can be modeled.

Finally we need to mention the computational complexity. The number of registrations to be computed is $N(N-1)$. All the computations were done with $2.6 \mathrm{GHz}$ processors. One registration on one processor takes approximately one hour. The database consists of $N=64$ images. The procedure would take $4096 \mathrm{~h}$ (170 days) using only one processor. The computations were done using 18 processors simultaneously, which reduced the computation time to \pm 10 days.

\section{References}

[1] Statistical Parameter Mapping. http://www.fil.ion.ucl.ac.uk/spm/spm99.html

[2] A. Guimond, J. Meunier and J-P. Thirion. Average brain models: a convergence study. Technical Report 3731, INRIA, 1999.

[3] F. Maes, A. Collignon, D. Vandermeulen, G. Marchal, and P. Suetens. Multimodality image registration by maximization of mutual information. IEEE Trans. Medical Imaging, 16(4):187-198, 1997.

[4] K. Van Leemput, F. Maes, D. Vandermeulen, P. Suetens. Automated model-based tissue classification of MR images of the brain. IEEE Trans. Medical Imaging, 18(10):897-908, 1999.

[5] E. D'Agostino, F. Maes, D. Vandermeulen, and P. Suetens. A viscous fluid model for multimodal non-rigid image registration using mutual information Medical Image Analysis, 7(4):565-575, 2003.

[6] C. Studholme. Simultaneous population based image alignment for template free spatial normalisation of brain anatomy. 2nd international workshop on biomedical image registration, June 2003, pp 81-90, LNCS 2717. 